\title{
Effects of Thymoquinone on Blood Parameters in Doxorubicin Cardiotoxicity
}

\author{
Deniz Yıldız Pehlivan ${ }^{*}$, Gülçin Durdağ ${ }^{1}$ \\ ${ }^{1} \dot{I}_{z m i r}$ Kâtip Çelebi University, Faculty of Medicine, Department of Physiology, İzmir, Turkey.
}

\begin{abstract}
Thymoquinone is known to alleviate cardiotoxic effects of doxorubicin. This study was conducted to investigate the effects of thymoquinone on biochemical parameter changes in cardiotoxicity caused by doxorubicin (DOX). 18 Wistar albino male rats were used in our study. The rats were divided into three groups as control, doxorubicin, and doxorubicin + thymoquinone $(D O X+T Q)$ group. The control group received one-time saline from the tail vein on the 5th day of the experiment. On the 5 th day of the experiment, the DOX group received a single dose of $45 \mathrm{mg} / \mathrm{kg}$ DOX from the tail vein. The DOX $+T Q$ group received $50 \mathrm{mg} / \mathrm{kg}$ thymoquinone by gavage for seven days and a single dose of $45 \mathrm{mg} / \mathrm{kg}$ DOX from the tail vein on the 5th day of the experiment. Rats were sacrificed under ketamine-xylazine anaesthesia and total blood samples were taken and biochemical parameters were evaluated on the last day of the experiment. According to the biochemical data, in DOX group in while the creatine kinase myocardial band (CK-MB), interleukin-6 (IL-6) interleukin-18 (IL-18), total oxidant status (TOS) and malondialdehyde (MDA) levels increased, the total antioxidant status (TAS) decreased compared to the control group. Compared to the DOX group, while the value of CK-MB, IL-6, IL-18, TOS, and MDA decreased, TAS increased in the DOX + TQ group. Results of our study shows that the deteriorative effects of doxorubicin on blood parameters can be corrected by thymoquinone.
\end{abstract}

Keywords: Doxorubicin, Thymoquinone, Cardiotoxicity.

\section{Introduction}

Doxorubicin (DOX or Adriamycin) was isolated for the first time in the 1960s and is among the most effective anticancer agents developed so far. It is classified in the cytotoxic antibiotics used in the treatment of many child and adult cancers such as haematological cancers, solid tumours, breast cancer, soft tissue sarcomas and lymphoma (1). However, acute, and chronic side effects

\footnotetext{
${ }^{*}$ Corresponding Author: Deniz Yıldız Pehlivan, Tel: +90 555557 8568, E-mail: deniz.yildiz.pehlivan@ikc.edu.tr, ORCID ID: 0000-0001-8127-2208.
} 
can occur in patients with cancer receiving DOX treatment. Acute side effects such as myelosuppression, nausea, vomiting, and arrhythmias are reversible and can be clinically controlled. Although DOX provides successful results in many cancer treatments, it causes irreversible cardiomyopathy as a chronic side effect and consequently heart failure. These chronic side effects have poor prognosis (2). Although it has good therapeutic potentials, the main limitation of DOX is due to its cardiotoxic side effects (3).

The mechanism of action of doxorubicin on tumour cells differs from the mechanisms that cause cardiotoxicity. The anti-malignant effects on tumour cells occur through the inhibition of the enzyme topoisomerase II, formation of reactive oxygen species and, consequently, DNA damage. In the mechanism that causes cardiotoxicity, it increases the reactive oxygen species (ROS) and triggers oxidative stress and lipid peroxidation and causes a decrease in the level of sulfhydryl groups and antioxidants (3).

There are many mechanisms that are thought to be responsible for the damage caused by DOX in the heart. Among them, various mechanisms have been proposed such as oxidative stress, myofibrillary disruption, intracellular calcium regulation disruption, apoptosis $(1,4)$. Decrease in antioxidant and sulfhydryl groups and increase in reactive oxygen species and lipid peroxidation shows that oxidative stress is crucial in DOXinduced cardiotoxicity (5-7). We used thymoquinone (TQ), an antioxidant substance that can be effective in preventing DOX cardiotoxicity, in this study.

Although many antioxidant substances have been used to prevent DOX cardiotoxicity, studies are ongoing to find the most ideal substance. In this study, thymoquinone, a phytochemical compound found in the oil of the plant nigella sativa was used to prevent DOX cardiotoxicity. Many properties of TQ (2-isopropyl-5-methyl-1,4-benzoquinone),

the most important bioactive component found in the essential oil of nigella sativa seed, have been reported. It has been reported in conducted studies that nigella sativa and its components have anti-carcinogenic, antitumour, anti-ulcerogenic, anti-bacterial, antiinflammatory and analgesic, antioxidant, hypoglycaemic and immune-enhancing effects (8). The protective effects of TQ have been reported as a "protective agent" by examining the toxicity caused by cyclosporine A (9), isoproterenol (10, 11), cyclophosphamide (12), and in ischemiareperfusion models (13-15).

The fact that DOX has exceptionally good therapeutic potential makes it indispensable in cancer treatments. However, the use of this drug has been limited because of its most important side effect, cardiotoxicity. In this study, we investigated the effects of thymoquinone, which has an antioxidant feature, on blood parameters to reduce the toxic effects of DOX. In this way, it is aimed to use DOX, which is restricted in use, with less side effects in chemotherapy.

\section{Materials and Methods}

Experimental Animals

This study was approved by Celâl Bayar University (CBU) Faculty of Medicine Local Ethics Committee of Experimental Animal use with the decision dated 09/06/2015 and numbered 77.637.435-51. 18 adult Wistar albino rats of 200-300 grams of each were 
used in the study. Experimental animals were obtained from CBU Experimental Animals Practice and Research Centre and kept in the same laboratory conditions during the study. During the experiment, they were kept in a 12-hour light-12-hour dark cycle and provided access to standard water and food (ad libitum nutrition). The rats were housed in the laboratory, which averaged $22 \pm 2{ }^{\circ} \mathrm{C}$ room temperature and $40 \pm 20 \%$ humidification. All experiments were carried out in accordance with the rules of Animal Ethics Committee and Welfare of the Experimental Animals.

18 rats were divided into 3 groups. There were 6 rats in each group. The DOX + TQ group received $50 \mathrm{mg} / \mathrm{kg}$ TQ with gavage for seven days and a single dose of $45 \mathrm{mg} / \mathrm{kg}$ DOX from the tail vein on the 5th day of the experiment. The control group received saline from tail vein on the 5th day of the experiment. The DOX group received a single dose of $45 \mathrm{mg} / \mathrm{kg} \operatorname{DOX}(6,7)$ from the tail vein on the 5 th day of the experiment. Rats were sacrificed on the last day of the experiment, their total blood was collected and processed for the biochemical parameters. The experiment schedule is shown in figure 1 .

\begin{tabular}{|c|c|c|c|c|c|c|c|c|}
\hline & 1st day & 2nd day & 3rd day & 4th day & 5th day & 6th day & 7th day & \multirow{4}{*}{ 8th day } \\
\hline $\begin{array}{l}\text { Control } \\
\operatorname{group}(n=6)\end{array}$ & & \multicolumn{6}{|c|}{$\begin{array}{l}\text { saline } \\
\text { infusion }\end{array}$} & \\
\hline $\begin{array}{l}\text { DOX group } \\
(n=6)\end{array}$ & & \multicolumn{6}{|c|}{$\begin{array}{l}\text { DOX } \\
\text { Infusion }\end{array}$} & \\
\hline $\begin{array}{l}\mathrm{DOX}+\mathrm{TQ} \\
\operatorname{group}(\mathrm{n}=6)\end{array}$ & & & Receiv & TQ for $\mathrm{s}$ & $\begin{array}{l}\text { DOX } \\
\text { infusion } \\
\text { en days }\end{array}$ & & & \\
\hline
\end{tabular}

Figure 1: Experiment Schedule. TQ; thymoquinone, DOX; doxorubicin.

Chemicals

Thymoquinone and doxorubicin hydrochloride were obtained from SigmaAldrich, $0.9 \% \mathrm{NaCl}$ from pharmacy, ketamine and xylazine from $\mathrm{CBU}$ Experimental Animal Research and Practice Centre.

Anaesthesia and Sampling

Ketamine (100 mg / kg) and xylazine (10 mg $/ \mathrm{kg}$ ) anaesthesia was applied to the rats on the last day of the experiment (8th day). Anaesthesia depth was evaluated with a foot pedal reflex. After deep anaesthesia, the thorax was opened with an abdominal incision and total blood was taken from their hearts. While plasma samples were obtained from the collected blood, erythrocytes were separated and stored at $-20{ }^{\circ} \mathrm{C}$ until analysis was performed. Superoxide dismutase (SOD) glutathione peroxidase (GPx) and catalase (CAT) antioxidant enzyme activities and MDA analyses were performed in erythrocyte hemolysate, while other parameters were measured in serum.

Biochemical Parameters

Nitric oxide (NO), hypoxia-induced factor-1 $\alpha(\mathrm{HIF}-1 \alpha), \mathrm{CK}-\mathrm{MB}, \mathrm{IL}-1, \mathrm{IL}-6$, and IL-18 levels were measured by ELISA tests. Analysis of total oxidant and antioxidant status were measured by TAS and TOS 
technique developed by Erel (16) (17). MDA analysis, on the other hand, determined with the method reported by Yoshioka et al. (18), Erythrocyte CAT, SOD and GPx activities were determined by the method described by Nadif et al. (19).

\section{Statistical analysis}

SPSS 22.0 (Statistical Package for Social Sciences) statistics program was used for statistical evaluations. The evaluation of the data was determined by one-way analysis of variance (ANOVA). Tukey test, which is one of the multiple comparison (post hoc) tests, was used to determine which groups were statistically different. Mean and standard deviations of the measurements obtained from all subjects and calculated variables were found. $\mathrm{p}<0.05, \mathrm{p}<0.01$ were accepted as statistically significant.

\section{Results}

The results of the biochemical parameters and statistical evaluations measured in the blood samples taken from the groups are shown in Table 1.

Table 1: Serum biochemical parameters (mean \pm SD: mean \pm standard deviation).

\begin{tabular}{|c|c|c|c|}
\hline & $\begin{array}{c}\text { Control } \\
(n=6) \\
\text { Mean } \pm \text { SD }\end{array}$ & $\begin{array}{c}\text { DOX } \\
(n=6) \\
\text { Mean } \pm \text { SD }\end{array}$ & $\begin{array}{c}\text { DOX+TQ } \\
(n=6) \\
\text { Mean } \pm \text { SD }\end{array}$ \\
\hline 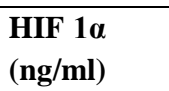 & $3.51 \pm 0.17$ & $3.81 \pm 0.63$ & $3.22 \pm 0.64$ \\
\hline $\begin{array}{l}\text { IL-1 } \\
(\mathrm{pg} / \mathrm{ml})\end{array}$ & $72.18 \pm 11.08$ & $81.08 \pm 13.76$ & $69.78 \pm 10.02$ \\
\hline $\begin{array}{l}\text { SOD } \\
\text { (U/mg Hb) }\end{array}$ & $1.86 \pm 0.82$ & $1.22 \pm 0.37$ & $1.79 \pm 0.54$ \\
\hline $\begin{array}{l}\text { GPx } \\
\text { (U/mg Hb) }\end{array}$ & $1.80 \pm 0.95$ & $1.45 \pm 0.33$ & $1.83 \pm 0.65$ \\
\hline $\begin{array}{l}\text { NO } \\
(\mu \mathrm{mol} / \mathrm{L})\end{array}$ & $61.42 \pm 11.56$ & $75.71 \pm 18.07$ & $64.50 \pm 6.86$ \\
\hline $\begin{array}{l}\text { CAT } \\
(k / g \text { Hb) }\end{array}$ & $45.70 \pm 29.32$ & $35.66 \pm 8.04$ & $44.22 \pm 22.84$ \\
\hline
\end{tabular}

Effect of Thymoquinone on Myocyte Damage Marker

As shown in Figure 2 (a), the serum level of CK-MB, an important marker of myocardial damage, increased significantly in the DOX group compared to the control group ( $\mathrm{p}$ $<0.05)$. However, when compared to the DOX group, the CK-MB level of the DOX + TQ group decreased significantly ( $\mathrm{p}<0.05)$. Effects of Thymoquinone on Pro- inflammatory Cytokines

Serum levels of pro-inflammatory cytokines IL-6 and IL-18 are shown in Figure 2 (b) and (c). Compared with the control group, there was a significant increase in both IL-6 (p $<0.05)$ and IL-18 ( $\mathrm{p}<0.01)$ levels in the DOX group. On the other hand, in the DOX + TQ group, there was a decrease in both IL-6 and IL-18 levels compared to the group given only DOX ( $\mathrm{p}<0.05)$. 

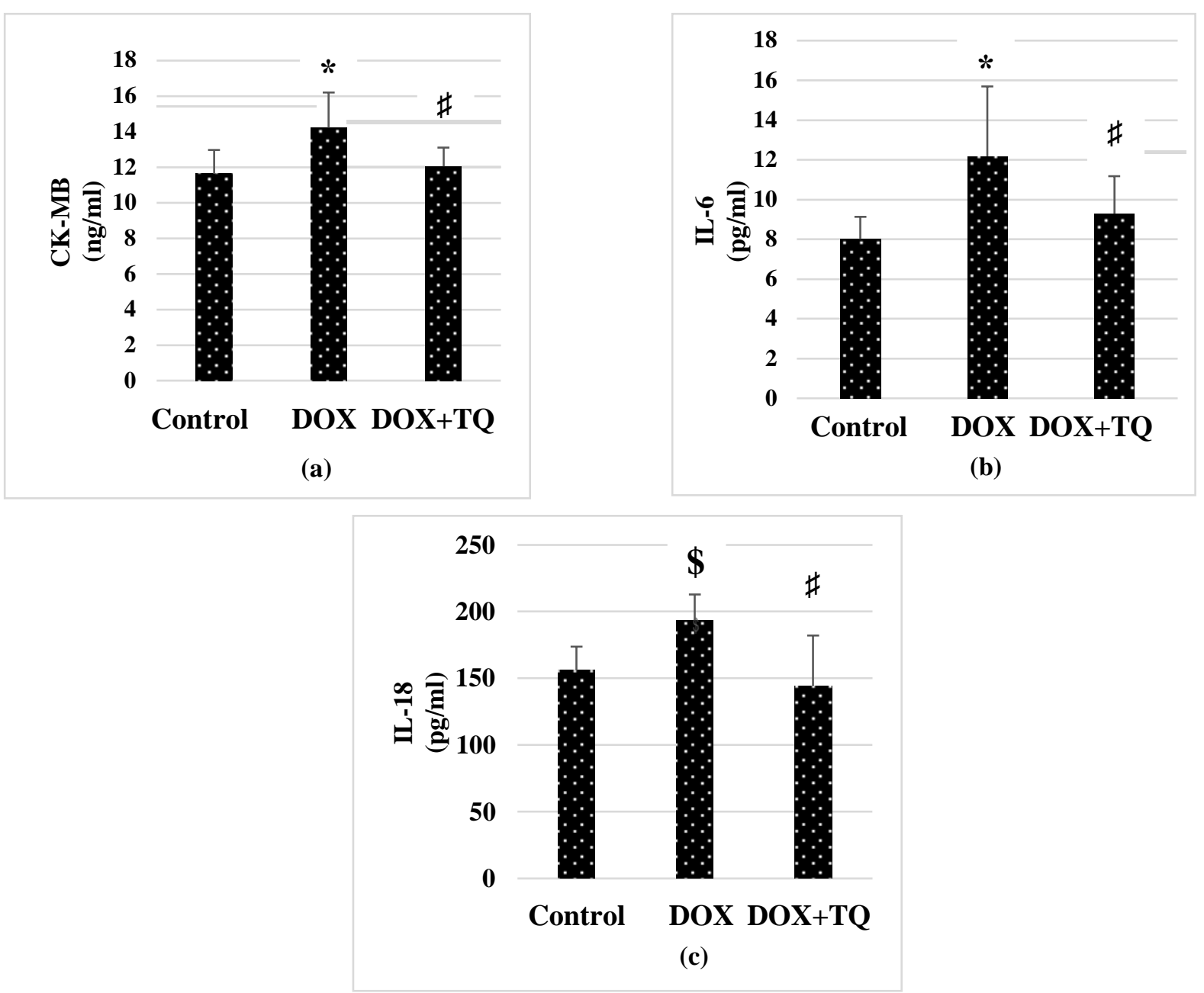

Figure 2: The effect of thymoquinone on serum CK-MB, IL-6, and IL-18 levels. *: Significant compared to the control group ( $\mathrm{p}<0.05$ ), \$: Significant compared to the control group ( $<<0.01$ ), \#: Significant compared to the DOX group ( $\mathrm{p}$ $<0.05)$.

Effect of Thymoquinone on Total Antioxidant and Total Oxidant Levels

TAS and TOS were analysed in serum from all experimental groups. As shown in Figure 3 (a) and (b), while the TAS decreased significantly ( $p<0.01$ ), the TOS increased significantly $(p<0.05)$ in the DOX group compared to the control group. While the TOS decreased ( $\mathrm{p}<0.05)$, the TAS increased
( $\mathrm{p}<0.05)$, when the DOX + TQ group compared to the DOX group.

A significant increase in the level of MDA, the end product of lipid peroxidation, was observed in the DOX group compared to control group ( $\mathrm{p}<0.01)$. The serum MDA levels decreased in the DOX + TQ group compared to the DOX group ( $\mathrm{p}<0.05)$, [Figure 3 (c)]. 

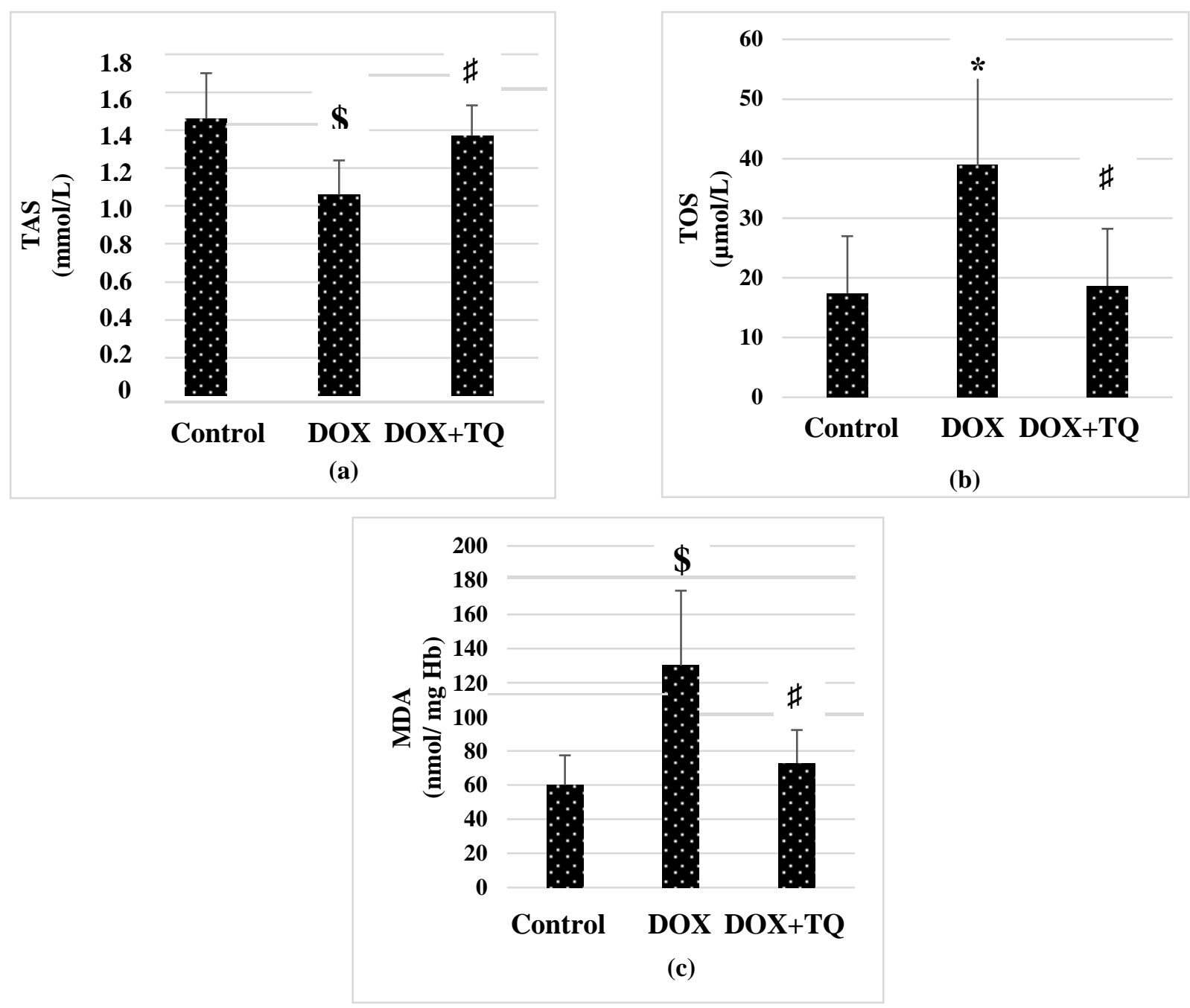

Figure 3: Effect of thymoquinone on serum TOS, TAS and MDA levels. *: Significant compared to the control group ( $\mathrm{p}<0.05)$, \$: Significant compared to the control group ( $\mathrm{p}<0.01$ ), \#: Significant compared to the DOX group ( $\mathrm{p}<0.05)$.

\section{Discussion}

This study set out with the aim of assessing the importance of thymoquinone in preventing the crucial side effects of doxorubicin. Doxorubicin is a broadspectrum anticancer agent used in many cancer treatments such as Hodgkin lymphoma, bladder, breast, stomach, lung, ovary, thyroid cancers, and soft tissue sarcomas. It has long been known that the most important side effect is cardiotoxicity, and its use has been limited due to this side effect. CK-MB, which is one of the enzymes that leak into the bloodstream when cardiac tissue damage occurs, is used as a cardiac marker in the diagnosis of cardiotoxicity (3). CK-MB is a plasma cardiac biomarker that helps in the laboratory diagnosis of heart attack as well.

DOX administration causes damage to the myocardial cell membrane or increase in permeability of the cell membrane resulting in CK-MB leakage into the blood (3). In our study, we revealed that CK-MB, an important 
cardiac marker and showing cardiac damage in the acute period, increased significantly only in the group receiving DOX. Compared to the DOX group, we found that the level of this marker in the DOX + TQ group significantly decreased in serum and approached the control group. Previous studies confirm our study results. It has been reported that $\mathrm{CK}-\mathrm{MB}$ increase due to DOX (3), cyclophosphamide (12), ischemia reperfusion (14) and streptozotocin-induced diabetes (20) decreased significantly with TQ. This might be due to the high antioxidant property of TQ, which reduces the heart muscle myofibril damage caused by DOX and consequently preventing $\mathrm{CK}-\mathrm{MB}$ isoenzyme leakage from cardiac tissue to blood. IL-18, a cytokine synthesized by Kupffer cells, has been reported to activate macrophages. In patients with acute myocardial infarction (MI) it has been reported to be activated the $\mathrm{T}$ cells and macrophages. It is assumed that IL-18 attend in the development of myocardial dysfunction via activation of immune cells. In one study, plasma IL-18 concentrations were found to be significantly higher in acute MI patients than in control patients. According to the results of the study, it was commented that high IL-18 concentration may be a new indicator of heart damage in the development of acute MI (21).

Production of reactive oxygen species (ROS) originating from doxorubicin usage causes oxidative stress and consequently cardiomyopathy (22). ROS activates proinflammatory cytokines. Compared to the control group, we found that the levels of IL6 and IL-18, which are pro-inflammatory cytokines, increased significantly in the DOX group. We showed that the levels of IL-6 and
IL-18 cytokines decreased in the DOX + TQ group. It was reported in previous studies that increase in the level of DOX-induced IL-2 (3) and the increase in the level of IL- 6 in diabetes-induced cardiomyopathy (20) were prevented by TQ. We think that TQ prevents the increase in pro-inflammatory cytokine levels by preventing the increase in ROS level (by neutralizing ROS).

Increased reactive oxygen species from DOX usage cause oxidative stress and consequently lipid peroxidation. MDA occurs because of lipid peroxidation and is an important indicator of damage caused by free radicals. Free radicals result in peroxidation of membrane phospholipids, causing changes in membrane permeability. DOX disrupts membrane functions by increasing free radicals and causes cardiotoxicity. In this study, a significant increase in the level of MDA occurred in the group receiving DOX. MDA levels decreased in the group receiving thymoquinone. In previous studies, it was reported that the increase of MDA in cardiotoxicity caused by DOX (3), isoproterenol (11), ethanol (23), carbon

tetrachloride (24), and diabetes (20) decreased by TQ. TQ may reduce oxidative stress and peroxidation of lipid membranes by neutralizing reactive oxygen species with its antioxidant properties.

DOX administration leads to increased ROS production in the body, cell dysfunction and impairment of cell membrane integrity. There are several antioxidant mechanisms in living organisms. The antioxidant system contains antioxidant enzymes and non-enzymatic antioxidants. Oxidant and antioxidant system balance is meticulously protected in the organism. When oxidants increase and antioxidants decrease, oxidative damage 
occurs in biological structures. The term "oxidative stress" refers to the deterioration of the pro-oxidant/pro-antioxidant balance in the body in favour of pro-oxidants. Since individual measurement of antioxidant levels is time consuming and expensive, all antioxidant levels should be measured in the evaluation of the oxidant status in vivo. Total oxidant status and total antioxidant status are particularly important in terms of evaluating the stress of the body. TOS and TAS parameters are a combination of all oxidant and antioxidant parameters such as MDA, glutathione peroxidase and catalase (25). In this study, we used the TAS and TOS technique developed by Erel to determine oxidant and antioxidant levels (17).

Compared to the control group, we found that TOS increased significantly and TAS decreased only in the group receiving DOX. While TAS was statistically increased in the group receiving TQ, TOS decreased. The balance deteriorated in favour of oxidants because of DOX administration was reversed by TQ. Our study results show that TQ is an important bioactive component that can play a role in maintaining the DOX-induced deteriorating oxidant-antioxidant balance. Our cells have various adaptation mechanisms to protect itself against oxygen deprivation. Cellular responses to hypoxia are mediated by hypoxia-induced factor-1 (HIF1). HIF-1 $\alpha$ provides transcription of proteins that respond cellularly to hypoxia (26). HIF$1 \alpha$ activation plays an important role in cellular protection and metabolic changes from the outcomes of oxygen deprivation during myocardial ischemia. An increase in HIF-l $\alpha$ level has been reported to be one of the first adaptive responses at the molecular level in myocardial ischemia (27). The expression of HIF-1 $\alpha$ was also previously report to increase with the progression of heart failure in rats with myocardial infarction or hamsters with cardiomyopathy. The level of transcription factor HIF-l $\alpha$ has been showed to increase significantly in the group receiving DOX compared to the control group (28). In other study have been reported no to be change in HIF-1 $\alpha$ expression in DOX-treated mouse heart (29). In our study, the HIF- $1 \alpha$ level measured in rat blood sample increased in the group treated with DOX. Compared to the DOX group, the level of HIF-1 $\alpha$ decreased in the group receiving DOX $+\mathrm{TQ}$. However, these differences were not statistically significant. $\mathrm{NO}$ is a free radical that has been play role in the aetiology of doxorubicin cardiotoxicity. In one study, DOX therapy has been shown to significantly increase plasma NO concentration. According to the results of the study, there was a relationship between plasmatic NO levels and histopathological myocardial damage. Therefore, it has been suggested that plasmatic NO levels can be used as a biomarker for DOX-induced myocardial damage (30). Fogli et al. had suggested that there is a decrease in cardiac contractility due to overproduction of reactive oxygen radicals and $\mathrm{NO}$, and the disruption in NO synthesis due to anthracyclines plays an important role in cardiotoxicity (31). In our study, serum NO concentrations of rats treated with DOX increased compared to the control group. The serum NO level of the rats receiving TQ decreased compared to the DOX group. However, these findings were not statistically significant.

In conclusion, the present study has gone some way towards enhancing our understanding of the effects of thymoquinone 
in the prevention of DOX-induced cardiotoxicity in rats. Further research is needed to determine the effectiveness of thymoquinone more clearly.

\section{Conflicts of Interest}

The authors declare that they have no conflicts of interest. The authors alone are responsible for the content and writing of this paper.

\section{Acknowledgement}

This study was supported by Scientific Research Fund of İzmir Kâtip Çelebi University. Project Number: 2015- ÖNPTIPF-0016.

\section{References}

1. Raj S, Franco VI, Lipshultz SE. Anthracycline-induced cardiotoxicity: A review of pathophysiology, diagnosis, and treatment. Current Treatment Options in Cardiovascular Medicine. Current Science Inc. 2014; 16: 315-28.

2. Singal PK, Li T, Kumar D, et al. Adriamycininduced heart failure: mechanism and modulation. Molecular and Cellular Biochemistry. 2000; 207: 77 85.

3. Alam MF, Khan G, Safhi MM, et al. Thymoquinone ameliorates doxorubicin-induced cardiotoxicity in swiss albino mice by modulating oxidative damage and cellular inflammation. Cardiology Research and Practice. 2018; 1-6.

4. Takemura G, Fujiwara H. Doxorubicininduced cardiomyopathy. From the cardiotoxic mechanisms to management. Progress in Cardiovascular Diseases. 2007; 49(5): 330-52.

5. Chatterjee $\mathrm{K}$, Zhang J, Honbo N, et al. Doxorubicin cardiomyopathy. Cardiology. 2010; 115: 155-62.

6. Oz E, Erbaş D, Sürücü HS, et al. Prevention of doxorubicin-induced cardiotoxicity by melatonin. Molecular and Cellular Biochemistry. 2006; 282: 317.
7. $\mathrm{Oz} \mathrm{E}$, İlhan MN. Effects of melatonin in reducing the toxic effects of doxorubicin. Molecular and Cellular Biochemistry. 2006; 286: 11-5.

8. Bacak Güllü E, Avcı G. Timokinon: Nigella Sativa'nın biyoaktif komponenti. Veterinary Journal. 2013; 6(1): 51-61.

9. Uz E, Uz B, Selcoki Y, et al. Cardioprotective effects of nigella sativa oil on cyclosporine A-induced cardiotoxicity in rats. Basic \& Clinical Pharmacology \& Toxicology. 2008; 103: 574-80.

10. Randhawa MA, Alghamdi MS, Maulik SK. The effect of thymoquinone, an active component of nigella sativa, on isoproterenol induced myocardial injury. Pak J Pharm Sci. 2013; 26(6): 1215-9.

11. Ojha S, Azimullah S, Mohanraj R, et al. Thymoquinone protects against myocardial ischemic injury by mitigating oxidative stress and inflammation. Evidence-Based Complementary and Alternative Medicine. 2015; 1-12.

12. Nagi MN, Al-Shabanah OA, Hafez MM, et al. Thymoquinone supplementation attenuates cyclophosphamide-induced cardiotoxicity in rats. Journal of Biochemical and Molecular Toxicology. 2011; 25(3): 135-42.

13. Seif AA. Nigella sativa attenuates myocardial ischemic reperfusion injury in rats. Journal of Physiology and Biochemistry. 2013; 69: 937-44.

14. Xiao J, Ke ZP, Shi Y, et al. The cardioprotective effect of thymoquinone on ischemiareperfusion injury in isolated rat heart via regulation of apoptosis and autophagy. Journal of Cellular Biochemistry. 2018; 119: 7212-8.

15. Aydin MS, Kocarslan A, Kocarslan S, et al. Thymoquinone protects end organs from abdominal aorta ischemia / reperfusion injury in a rat model. Braz J Cardiovasc Surg. 2015; 30(1): 77-83.

16. Erel O. A novel automated direct measurement method for total antioxidant capacity using a new generation, more stable ABTS radical cation. Clinical Biochemistry. 2004; 37: 277-85.

17. Erel O. A new automated colorimetric method for measuring total oxidant status. Clinical Biochemistry. 2005; 38: 1103-11.

18. Yoshioka T, Kawada K, Shimada T, et al. Lipid peroxidation in maternal and cord blood and protective mechanism against activated-oxygen toxicity in the blood. American Journal of Obstetrics and Gynecology. 1979; 135: 372-6. 
19. Nadif R, Bourgkard E, Dusch M, et al. Relations between occupational exposure to coal mine dusts, erythrocyte catalase and $\mathrm{Cu}++\mathrm{Zn}++$ superoxide dismutase activities, and the severity of coal workers' pneumoconiosis. Occupational and Environmental Medicine. 1998; 55: 533-40.

20. Atta MS, El-Far AH, Farrag FA, et al. Thymoquinone attenuates cardiomyopathy in streptozotocin-treated diabetic rats. Oxidative Medicine and Cellular Longevity. 2018; 1-10.

21. Seta Y, Kanda T, Tanaka T, et al. Interleukin 18 in acute myocardial infarction. Heart. 2000; 84: 668.

22. Zheng J, Lee HCM, Bin Sattar MM, et al. Cardioprotective effects of epigallocatechin-3-gallate against doxorubicin-induced cardiomyocyte injury. European Journal of Pharmacology. 2011; 652: 82-8.

23. Arslan SO, Gelir E, Armutcu F, et al. The protective effect of thymoquinone on ethanol-induced acute gastric damage in the rat. Nutrition Research. 2005; 25: 673-80.

24. Mansour MA. Protective effects of thymoquinone and desferrioxamine against hepatotoxicity of carbon tetrachloride in mice. Life Sciences. 2000; 66(26): 2583-91.

25. Yuluğ E, Turedi S, Karaguzel E, et al. The short term effects of resveratrol on ischemiareperfusion injury in rat testis. Journal of Pediatric
Surgery. 2014; 49: 484-9.

26. Spagnuolo RD, Recalcati S, Tacchini L, et al. Role of hypoxia-inducible factors in the dexrazoxanemediated of cardiomyocytes from doxorubicininduced toxicity. British Journal of Pharmacology. 2011;163: 299-312.

27. Zhao HX, Wang XL, Wang YH, et al. Attenuation of myocardial injury by postconditioning: role of hypoxia inducible factor- $1 \alpha$. Basic Res Cardiol. 2010; 105: 109-18.

28. Ichihara S, Yamada Y, Kawai Y, et al. Roles of oxidative stress and Akt signaling in doxorubicin cardiotoxicity. Biochemical and Biophysical Research Communications. 2007; 359: 27-33.

29. Soni H, Pandya G, Patel P, et al. Beneficial effects of carbon monoxide-releasing molecule-2 (CORM-2) on acute doxorubicin cardiotoxicity in mice: Role of oxidative stress and apoptosis. Toxicology and Applied Pharmacology. 2011; 253: 70-80.

30. Guerra J, De Jesus A, Santiago-Borrero P, et al. Plasma nitric oxide levels used as an indicator of doxorubicin-induced cardiotoxicity in rats. The Hematology Journal. 2005; 5: 584-8.

31. Fogli S, Nieri P, Breschi MC. The role of nitric oxide in anthracycline toxicity and prospects for pharmacologic prevention of cardiac damage. Faseb Journal. 2004; 18: 664-75. 